\title{
Simultaneous Measurement of One Dimensional Bending and Temperature Based on Mach-Zehnder Interferometer
}

\author{
Yongqin YU ${ }^{1,2,3^{*}}$, Yufeng ZHANG ${ }^{1,2,3}$, Zhilong OU ${ }^{1,2,3}$, Xue $\mathrm{CHEN}^{1,2,3}$, \\ Quandong HUANG ${ }^{1,2,3}$, and Shuangchen RUAN ${ }^{1,2,3}$
}

\author{
${ }^{1}$ College of Optoelectronic Engineering, Shenzhen University, Shenzhen, 518060, China \\ ${ }^{2}$ Shenzhen Key Laboratory of Laser Engineering, Shenzhen, 518060, China \\ ${ }^{3}$ Key Laboratory of Advanced Optical Precision Manufacturing Technology of Guangdong Higher Education Institutes, \\ Shenzhen University, Shenzhen, 518060, China
}

*Corresponding author: Yongqin YUＥ-mail: yuyq@szu.edu.cn

\begin{abstract}
A simple and compact optical fiber directional bending vector sensor with simultaneous measurement of temperature based on the Mach-Zehnder interferometer (MZI) is proposed and experimentally demonstrated. The device consists of a piece of photonic crystal fiber (PCF) sandwiched between two single mode fibers (SMFs) with a lateral offset splicing. It shows the capacity for recognizing positive and negative directions. Within a curvature range of $-7.13 \mathrm{~m}^{-1}$ to $7.13 \mathrm{~m}^{-1}$, the bending sensitivities of two resonant dips with opposite fiber orientations are obtained to be $0.484 \mathrm{~nm} / \mathrm{m}^{-1}$ and $0.246 \mathrm{~nm} / \mathrm{m}^{-1}$, respectively. This simple MZI is formed by invoking interference between $\mathrm{LP}_{01}$ and $\mathrm{LP}_{21}$ core modes, which leads to that the sensor is not sensitive to ambient refractive index (ARI). The temperature sensitivity has also been investigated. Two dips have obviously different sensitivities on the temperature and bending, so two parameters of both curvature and temperature can be distinguished and measured simultaneously by constructing a matrix and using one simple model interferometer.
\end{abstract}

Keywords: Fiber optics sensors, microstructured fiber, temperature sensor, curvature sensor

Citation: Yongqin YU, Yufeng ZHANG, Zhilong OU, Xue CHEN, Quandong HUANG, and Shuangchen RUAN, "Simultaneous Measurement of One Dimensional Bending and Temperature Based on Mach-Zehnder Interferometer," Photonic Sensors, 2015, 5(4): 376-384.

\section{Introduction}

Curvature is one of important physical parameters which are essential to monitor in some applications such as structural deformation, structural health monitoring, intelligent artificial limb, and mechanical engineering. Fiber optic sensors have got a growing interest due to their well-known superior advantages over conventional sensors, such as compact and integrated structure, immunity to electromagnetic interference, high sensitivity, in situ measurements, and potentially low cost $[1,2]$. Up to now, the curvature characteristics of fiber sensors have been studied in many works, for example, fiber grating-based sensors using the long period fiber grating (LPFG) [1], fiber Bragg grating (FBG) [2], tilted FBG [3], Mach-Zehnder interferometers (MZIs) based on fiber lateral-offset splicing [4], and two identical fused fiber tapers [5] or multimode fiber combined

Received: 22 May 2015 / Revised: 10 September 2015

(C) The Author(s) 2015. This article is published with open access at Springerlink.com

DOI: $10.1007 / \mathrm{s} 13320-015-0264-\mathrm{x}$

Article type: Regular 
with an LPFG [6]. Usually, the fabricating process of the grating-based curvature sensors is relatively complicated and high power laser is usually needed $[2,6]$. In this regard, the MZI-based curvature sensors as alternatives have attracted lots of attentions. Generally, the structure of the MZI-based curvature sensor is relatively simple and easy to fabricate. Since the curvature applied on the MZI affects the two arms differently, the optical path difference will be changed to result in the wavelength shift of the interference transmission spectrum. So the structure of the MZI can be used to sense the applied curvature. Up to now, there were different types of devices widely proposed and studied [4-6]. But there were few reports on the direction recognition bending sensing with the MZI in the past few years. Until 2012, Zhang et al. presented a fiber-optic directional bending sensor based on MZI exploiting lateral offset and up taper in single mode fibers (SMF) [7]. For a curvature range from $-3 \mathrm{~m}^{-1}$ to $3 \mathrm{~m}^{-1}$, the bending sensitivities at wavelengths of $1463.86 \mathrm{~nm}$ and $1548.41 \mathrm{~nm}$ reached $11.987 \mathrm{~nm} / \mathrm{m}^{-1}$ and $8.697 \mathrm{~nm} / \mathrm{m}^{-1}$, respectively. However, the MZI-based bending device mentioned above usually excites the cladding mode, which is sensitive to ambient refractive index (ARI) variation, so that the cross sensitivity of ARI is a critical disadvantage. In 2013, Ou from our research group [8] reported a compact optical fiber directional bending vector sensor based on the MZI which consists of a piece of seven-core photonic crystal fiber (PCF) sandwiched between two SMFs. For a curvature range of $-7.05 \mathrm{~m}^{-1}$ to $7.05 \mathrm{~m}^{-1}$, the bending sensitivities of two resonant central wavelengths were $1.232 \mathrm{~nm} / \mathrm{m}^{-1}$ and $1.174 \mathrm{~nm} / \mathrm{m}^{-1}$, respectively. However, the crosstalk of temperature in the curvature measurement for the application is necessary to be eliminated.

In this paper, we demonstrate a simple and compact fiber sensor based on the MZI, which has the capability of simultaneous measurement of one dimensional bending vector and temperature. The MZI is simply fabricated by splicing a section of multimode PCF to single mode fibers (SMFs) with a lateral offset splicing for exciting the higher order core modes. A dominant interference pattern is generated by $\mathrm{LP}_{01}$ and higher order $\mathrm{LP}_{21}$ mode in the core. Thus, this sensor is not sensitive to the ARI and can sense the bending vector in one dimension. Meanwhile, using the obvious different sensing characteristics of two transmission dips on the temperature and bending, two parameters can be simultaneously distinguished and measured by constructing a matrix consisting of the curvature and temperature sensitivities.

\section{Sensor fabrication and operation principle}

The cross section of PCF captured by scanning electron micrograph (SEM) is shown in the inset of Fig. 1. The PCF has a micro-structured cladding formed by 8 layered air holes in silica. The diameter of the silica core $a$, the air holes $d$, the outer cladding, and the pitch of air holes $\Lambda$ are $5.0 \mu \mathrm{m}$, $4.55 \mu \mathrm{m}, 133 \mu \mathrm{m}$, and $5.45 \mu \mathrm{m}$, respectively. The air filling fraction $d / \Lambda$ is obtained to be 0.83 . According $[9,10]$, when air-filling fraction is in the range of 0.45 to 0.65 , PCF supports dual-mode operation, while beyond 0.65 , PCF operates in multimode. Therefore, such a PCF used in our experiment can be considered to be a multimode fiber.

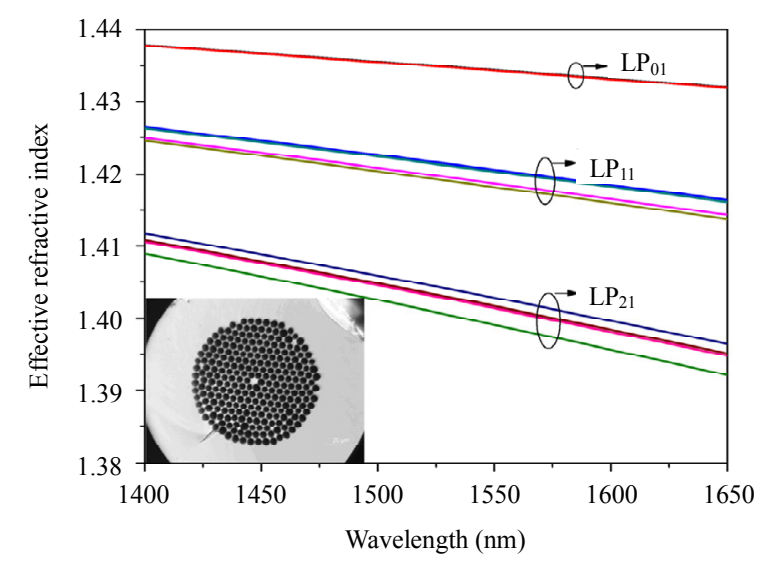

Fig. 1 Effective mode index versus wavelength for PCF. The inset is the cross section of PCF taken by SEM. 
The propagation property considering the material dispersion of silica given by the Sellmeier equation for PCF is carried out by using the full-vector finite element method (FEM, COMSOL Multiphysics). The relationship of simulated effective mode indices of $\mathrm{LP}_{01}, \mathrm{LP}_{11}$, and $\mathrm{LP}_{21}$ with wavelength is plotted in Fig. 1. The effective index difference of $\mathrm{LP}_{01}$ mode and $\mathrm{LP}_{21}$ mode at $1500 \mathrm{~nm}$ is calculated to be about 0.030 . The three mode bands are distinguished by the calculated mode field distribution in PCF as shown in Fig. 1. The mode profiles of $\mathrm{LP}_{01}, \mathrm{LP}_{11}$, and $\mathrm{LP}_{21}$ at $1500 \mathrm{~nm}$ of different orders are demonstrated in Figs. 2(a) and 2(b), Figs.2(c), 2(d), 2(e), and 2(f) and Figs. 2(g), 2(h), 2(i), and 2(j), respectively.

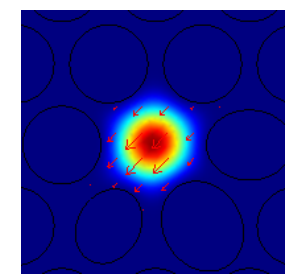

(a)

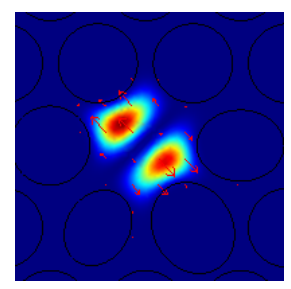

(f)

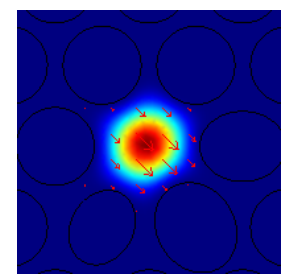

(b)

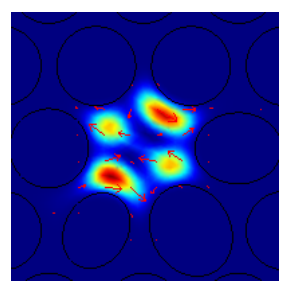

(g)

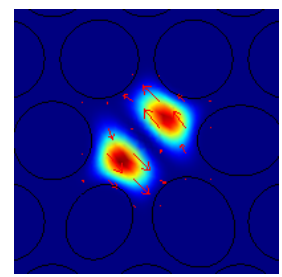

(c)

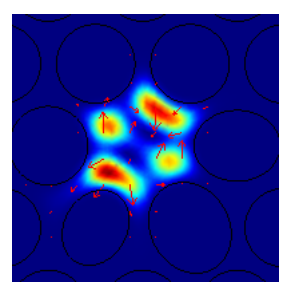

(h)

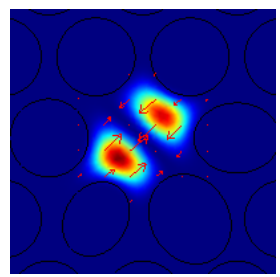

(d)

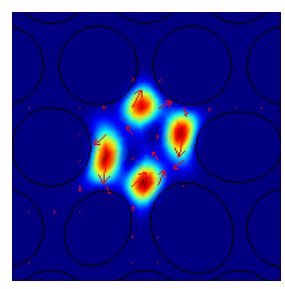

(i)

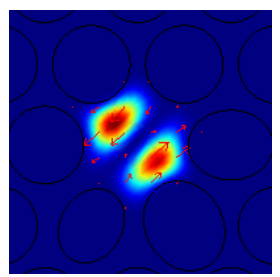

(e)

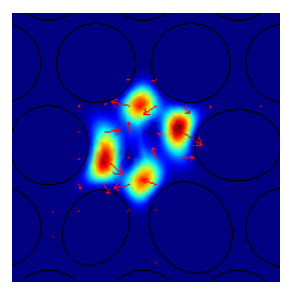

(j)

Fig. 2 Calculated mode field distribution for different modes of PCF at $1500 \mathrm{~nm}$ : (a) and (b): $\mathrm{LP}_{01}$, (c) (d) (e) and (f): $\mathrm{LP}_{11}$, (g) (h) (i) and (j): $\mathrm{LP}_{21}$ (arrows represent the amplitudes and directions of transverse electric fields).

The schematic structure of the PCF based MZI is shown in Fig. 3. To construct the MZI structure, an unjacketed PCF with a short length is spliced between two SMFs with a commercial fusion splicing machine (FITEL S175). The photograph of two splicing points of the PCF we used is shown in the insets of Fig. 3, captured by the optical microscope (Olympus BX51, Olympus Inc.). The left one is corresponding to the first splice point with lateral offset and the right one is the second splice point without lateral offset. From the early reports, we know that the higher order core modes can be excited by laterally core-offset splicing between the sensing fiber and lead fiber, so this strategy is also employed to construct the modal interferometer. At the first splice point, the lateral offset of PCF and SMF is set to be $1.9 \mu \mathrm{m}$ corresponding to the highest visibility of fringe pattern. Light is injected into the PCF from lead-in SMF and high-order core modes are excited at the first splice point of PCF because of the certain intentional lateral offset fusion splicing [9]. At the second splice point without lateral offset between PCF and SMF-28, the light of fundamental mode and high order modes are recoupled back to the core of SMF and results in the interference fringe.

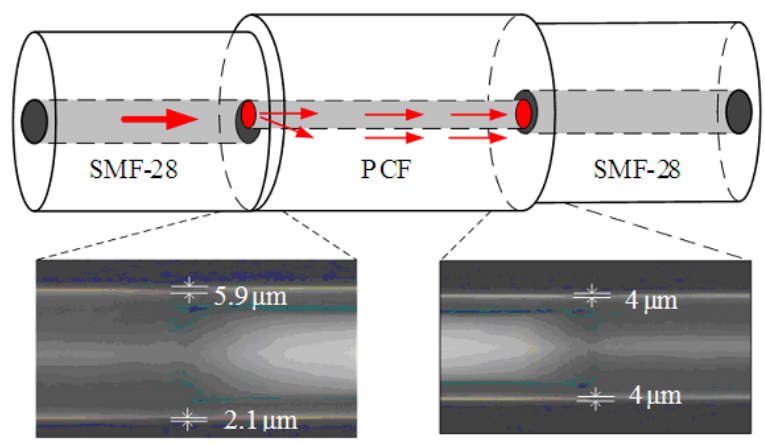

Fig. 3 Schematic diagram of the MZI with a section of PCF.

According to the interference theory, the difference in the effective refractive index of the 
different core modes that propagate along the PCF results in different optical path lengths of the interferometer arms. The transmission power as a function of the wavelength can be expressed as [11]

$$
I(\lambda)=I_{1}(\lambda)+I_{2}(\lambda)+2 \sqrt{I_{1}(\lambda) I_{2}(\lambda)} \cos \left(\frac{2 \pi \Delta n_{\mathrm{eff}} L}{\lambda}\right)
$$

where $I_{1}$ and $I_{2}$ are the powers of the two interferential core modes, $L$ is the length of the PCF, $\lambda$ is the operating wavelength, and $\Delta n_{\text {eff }}$ is the effective refractive index difference of two core modes. The accumulated phase difference between two modes can be expressed as [11]

$$
\phi=2 \pi \Delta n_{\text {eff }} L / \lambda \text {. }
$$

When the phase difference $\phi$ satisfies the resonate condition $\phi=(2 m+1) \pi$, a transmission resonate dip will appear at

$$
\lambda_{m}=2 \Delta n_{\text {eff }} L /(2 m+1)
$$

where $m$ is an integer. When the temperature of external environment varies or external curvature is applied on the structure of the MZI, the phase difference of two modes will be changed, which results in the wavelength shift of the interference transmission spectrum. So the structure of the MZI can be used to sense the applied parameters from ambient. The interference fringe spacing $\xi$ of the MZI can be expressed as $[12,13]$

$$
\xi=\frac{\lambda^{2}}{\Delta n_{\mathrm{eff}} \cdot L} .
$$

In the experiments, to analyze the mode interference, PCF MZIs are fabricated with the interference lengths of $L=4 \mathrm{~mm}$ and $L=13.8 \mathrm{~mm}$, respectively. The light source used for the transmission spectra is a broadband super luminescent light-emitting diode (SLED) optical source ranging from $1250 \mathrm{~nm}$ to $1700 \mathrm{~nm}$ (B\&A Technology SL3200, China), and an optical spectral analyzer (OSA) with $0.1 \mathrm{~nm}$ spectral resolution (YOKOGAWA AQ6370B) is used to detect the spectra of the MZIs. Figure4 shows the transmission spectra without bending of the structure of MZI for the interference lengths of $L=4 \mathrm{~mm}$ and $L=13.8 \mathrm{~mm}$, respectively. Strong interference pattern and good fringe visibility can be seen from Fig. 4, and the interference fringes for $L=13.8 \mathrm{~mm}$ become denser than that for $L=4 \mathrm{~mm}$. It is because the interference fringe spacing $\Lambda$ and the interference length $L$ have an inverse relationship, as revealed by (4). The maximum fringe extinction ratios (ER) of the interference resonance dips are $15 \mathrm{~dB}$ and $19 \mathrm{~dB}$, for $L=13.8 \mathrm{~mm}$ and $L=4 \mathrm{~mm}$, respectively. From Fig. 4, it can be seen the interference pattern is produced by the overlap of multiple inter-modal interference. The interference pattern is formed by the fundamental core mode and dominant high ordered mode, while the other weak mode has a modulation function on the interference pattern [14]. From Fig. 4, the

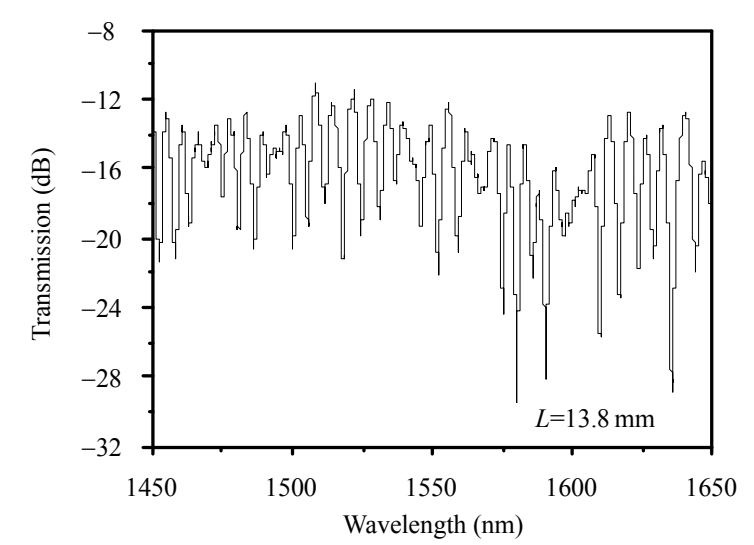

(a)

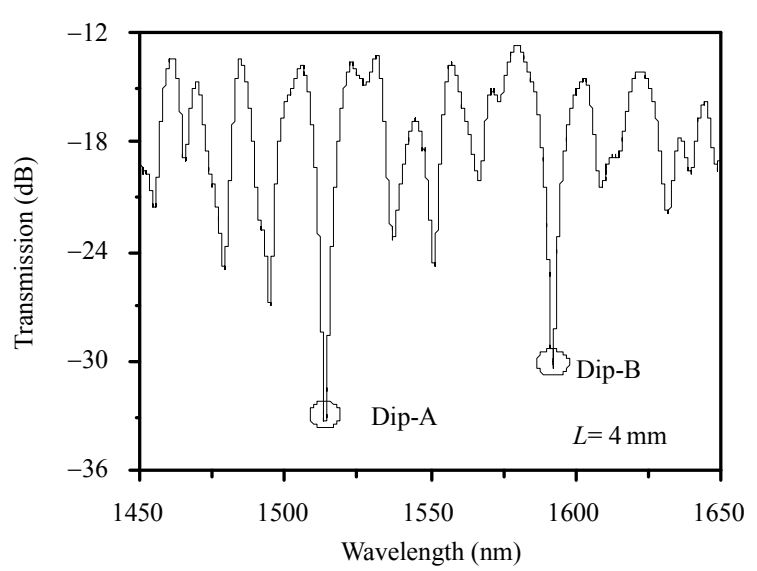

(b)

Fig. 4 Transmission spectra of the MZIs with different interference lengths: (a) $13.8 \mathrm{~mm}$ and (b) $4 \mathrm{~mm}$. 
interference fringe spacing of the MZI with $L=13.8 \mathrm{~mm}$ and $4 \mathrm{~mm}$ are $5.3 \mathrm{~nm}$ and $18.9 \mathrm{~nm}$ at $1500 \mathrm{~nm}$, respectively. Therefore, using (4), we can get the average effective mode refractive index difference between the core mode and the higher-order mode at around $1500 \mathrm{~nm}$, that is 0.0308 and 0.0299, respectively, which agrees well with the effective index difference 0.030 of $\mathrm{LP}_{01}$ mode and $\mathrm{LP}_{21}$ mode at $1500 \mathrm{~nm}$ calculated in theory by the FEM, as shown in Fig. 1. Therefore, the $\mathrm{LP}_{21}$ mode is confirmed to be the dominant high order core mode interfering with the fundamental core mode. The experimental value of $\Delta n_{\text {eff }}$ is close to the theoretical one above. The insertion loss (the minimum value of about $12 \mathrm{~dB}$ ) is a little higher, which is attributed to the lateral offset splicing and the mismatch of mode fields between SMF and PCF. From Fig. 4, the MZI with a PCF length of $L=4 \mathrm{~mm}$ has a larger free spectral range (FSR) due to its wider interference fringe spacing. So it is used to implement the experimental data to investigate the sensitivity to the temperature and curvature.

\section{Results and discussion}

Figure 5(a) shows the schematic diagram of the experimental setup for measuring the curvature characteristics of the structure of MZI. The broad-band SLED and OSA are used to connect to the MZI to monitor the transmission interference spectra as the curvature varies. The MZI under test is positioned at the middle of a section of SMF, and then two ends of the fiber are fixed at two graduated rotational fiber holders, respectively. Bending is applied to the MZI by moving one translation stage inward. The fiber is set to bend toward the $-Y$ axis direction and then is normally approximated and acted as an arc circle. The resulting applied curvature $(C)$ of the sensor can be defined as [1]

$$
C=2 d /\left(d^{2}+L^{2}\right)
$$

where $d$ is the bending displacement at the center of the MZI and $2 L$ is the distance between the two graduated rotational fiber holders. The fiber can be rotated coaxially by using two graduated rotational holders, so the rotated angle of the holders corresponds to the fiber orientation which can determine the directional bending characteristics of the MZI. Figure 5(b) shows the four fiber orientations used in the experiments. The fiber orientations of $0^{\circ}$ and $180^{\circ}$ are corresponding to the directions of the lateral offset of PCF and SMF. And those of $90^{\circ}$ and $270^{\circ}$ are perpendicular to the offset.

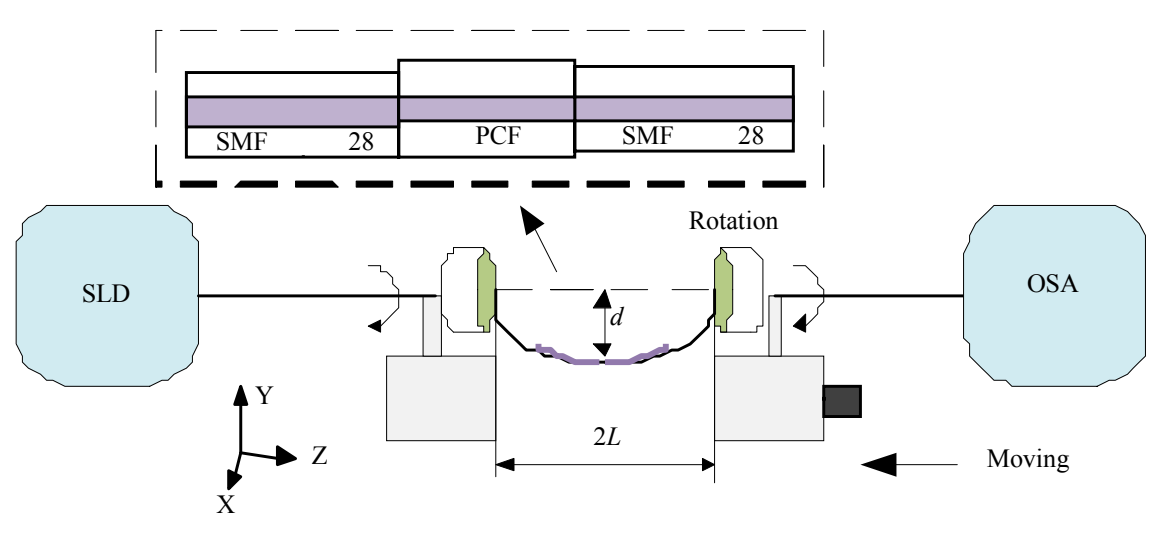

(a)
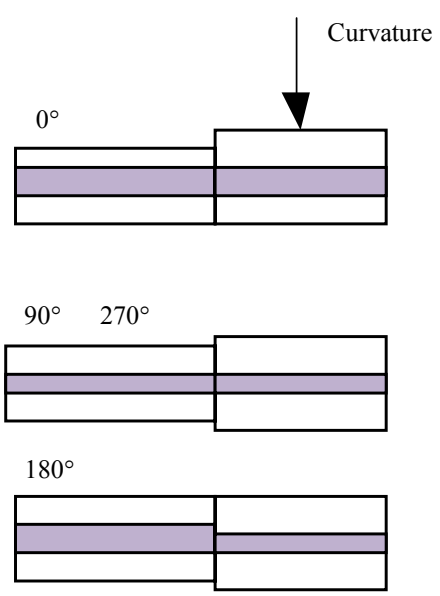

(b)

Fig. 5 Schematic diagram of the experimental setup for bending measurement and bending orientations: (a) schematic diagram of the experimental setup and (b) different fiber orientations.

The PCF MZI with a length of $4 \mathrm{~mm}$ is used to different axial rotation angles of the fiber at room record the response to variations of curvature for temperature. We have traced the transmission 


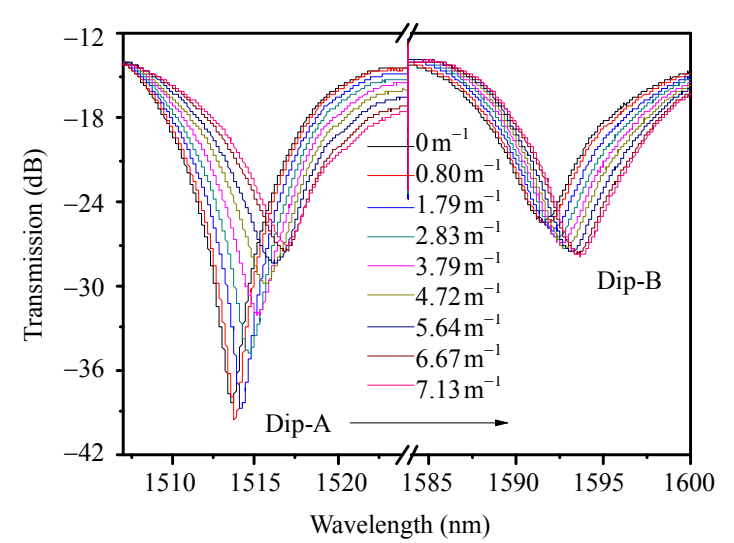

(a)

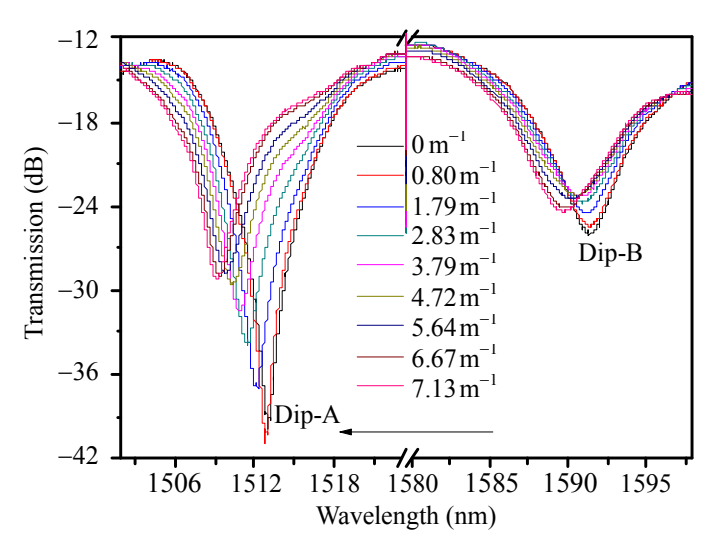

(b)

Fig. 6 Spectral responses of the MZI with an increase in the curvature for different axial orientation angles of the fiber: (a) $0^{\circ}$ and (b) $180^{\circ}$.

spectra of the MZI under various applied curvatures, especially the two dips marked by Dip-A and Dip-B, as shown in Fig.4(b). The spectral evolution of two dips under different curvatures for the fiber orientation of $0^{\circ}$ and $180^{\circ}$ are given in Figs. 6(a) and 6(b), respectively. It can be seen clearly that the MZI sensor shows a directional bending sensitivity corresponding to the opposite fiber orientations. When the MZI is located at $0^{\circ}$ orientation, the central wavelengths of resonant dips are both shifted to the longer wavelength as the applied curvature increases, whereas experience a blue shift for the case of $180^{\circ}$ orientation. As applied curvature on the PCF increases, the extinction ratio (ER) of the interferometer decreases accordingly due to the bending loss of different core mode re-coupling. It is clear that the ER of Dip-A is strongly decreased as the applied curvature increases both for the fiber orientation of $0^{\circ}$ and $180^{\circ}$, while that of Dip-B is weakly dependent on the curvature. Assuming the power distributed in the core mode and dominant high order mode are $I_{1}$ and $I_{2}$, respectively, the ER can be expressed [14] as

$$
\mathrm{ER}=10 \lg \left(\frac{1+\sqrt{\kappa I_{2} / I_{1}}}{1-\sqrt{\kappa I_{2} / I_{1}}}\right)^{2}
$$

where $\kappa$ is a constant, describing the coupling efficiency of the dominant high ordered core mode to the fundamental core mode, which is dependent on the fusion splice quality of the splice point. Hence, ER is related to the power ratio of $I_{2} / I_{1}$. With an increase in the curvature, the high order mode will experience bending loss while the power distributed in the fundamental core mode will keep almost unchanged. This will lead to a decrease in $I_{2} / I_{1}$, then the ER will change accordingly.

Figure 7(a) shows the wavelength shifts of two resonant dips, located initially at $1513 \mathrm{~nm}$ and $1591 \mathrm{~nm}$, plotted against applied curvature for inverse fiber orientations $\left(0^{\circ}\right.$ and $\left.180^{\circ}\right)$, respectively. It can be seen that the bending responses of the MZI show a directionally sensitive property and the wavelength shifts exhibit a strongly linear relationship with the $\mathrm{R}$-squared values all lying within 0.995 in a large curvature range from $-7.13 \mathrm{~m}^{-1}$ to $7.13 \mathrm{~m}^{-1}$. The bending sensitivities of the two dips are obtained to be $S_{C, A}=0.484 \mathrm{~nm} / \mathrm{m}^{-1}$ and $S_{C, B}=0.246 \mathrm{~nm} / \mathrm{m}^{-1}$ by linear fitting, respectively. Compared with the results reported before, the curvature sensor with the high bending sensitivities usually shows a small measurement range. For example, in 2013, a curvature fiber optic sensor using a two-core fiber [15] exhibited a sensitivity of $-137.87 \mathrm{~nm} / \mathrm{m}^{-1}$ for a small curvature ranging from 0 to $0.27 \mathrm{~m}^{-1}$. In 2011, Dong et al. [14] presented a temperature insensitive curvature sensor with one section of polarization maintaining photonic crystal fiber (PMPCF) by core-offsetting one splice joint of an SMF-PMPCF-SMF structure. The sensitivity of $2.826 \mathrm{~nm} / \mathrm{cm}^{-1}$ is obtained within a small curvature range from $0.125 \mathrm{~cm}$ to $0.333 \mathrm{~cm}^{-1}$. The dependence 
of wavelength shifts of Dip-A upon the applied curvature with the fiber orientation at $90^{\circ}$ and $270^{\circ}$ is also experimentally monitored, as shown in Fig. 7(b). The wavelength shift is insensitive to applied bending in the curvature range from 0 to $7.13 \mathrm{~m}^{-1}$, and the bending sensitivity is found no directional characteristic. It is due to that the structure of the MZI is symmetric in the $X$ axis direction, and thus the optical path difference (OPD) of the MZI with the applied curvature will almost be unchanged for the fiber orientations at $90^{\circ}$ and $270^{\circ}$, meanwhile resulting in the same attenuation wavelength shifts in the opposite direction [7]. It indicates that this structure of the MZI can work as a one-dimensional bending vector sensor.

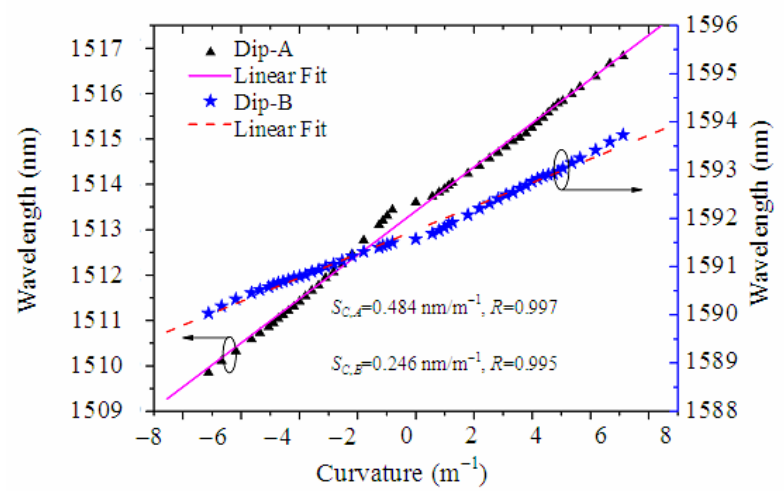

(a)

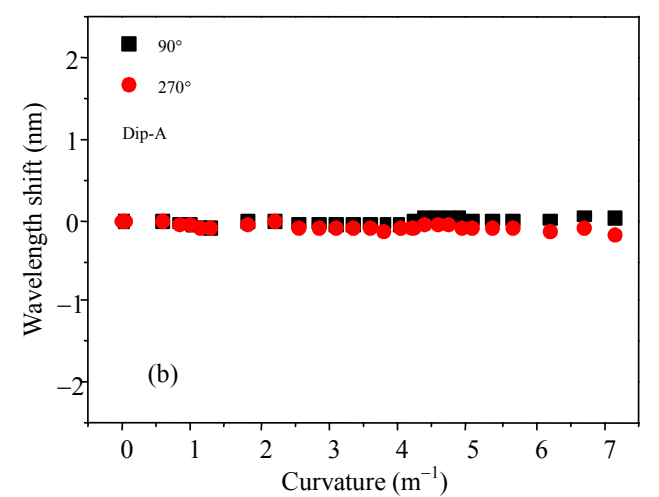

(b)

Fig. 7 Wavelength shifts of the MZI against applied curvature for different fiber orientations: (a) triangle: Dip-A $\left(0^{\circ}\right.$ and $\left.180^{\circ}\right)$; star: Dip-B $\left(0^{\circ}\right.$ and $\left.180^{\circ}\right)$ and (b) Dip-A $\left(90^{\circ}\right.$ and $\left.270^{\circ}\right)$.

The crosstalk of ARI and temperature for a bending sensor is a critical issue that should be taken into account. In our experiments, the temperature response of the MZI structure is investigated. The sensor structure is placed in a heater which controls the temperature from $30{ }^{\circ} \mathrm{C}$ to $150{ }^{\circ} \mathrm{C}$ with an interval step of $10{ }^{\circ} \mathrm{C}$. The linear relationship of wavelength shift with temperature is shown in Fig. 8(a). Dip-A and Dip-B are both linearly blue-shift as temperature increases and have different temperature sensitivities. Dip-B at longer wavelength has a lower sensitivity. By linear fitting, the temperature sensitivities at wavelengths of Dip-A and Dip-B are obtained to be $S_{T, A}=$ $-0.023 \mathrm{~nm} /{ }^{\circ} \mathrm{C}$ and $S_{T, B}=-0.016 \mathrm{~nm} /{ }^{\circ} \mathrm{C}$, respectively.

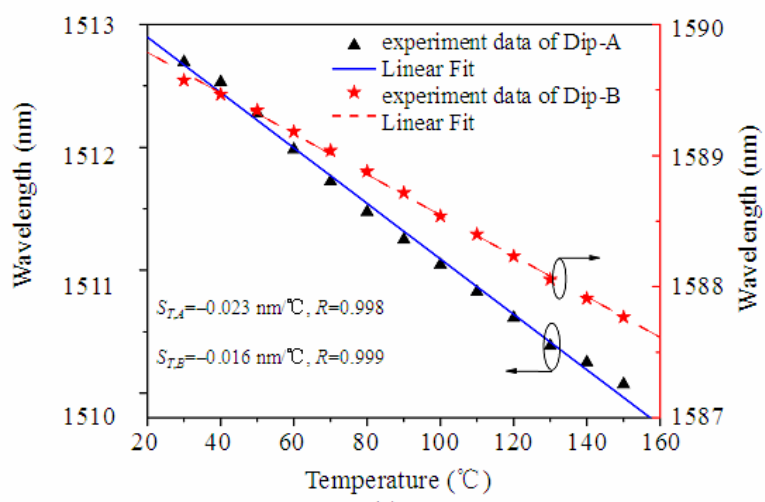

(a)

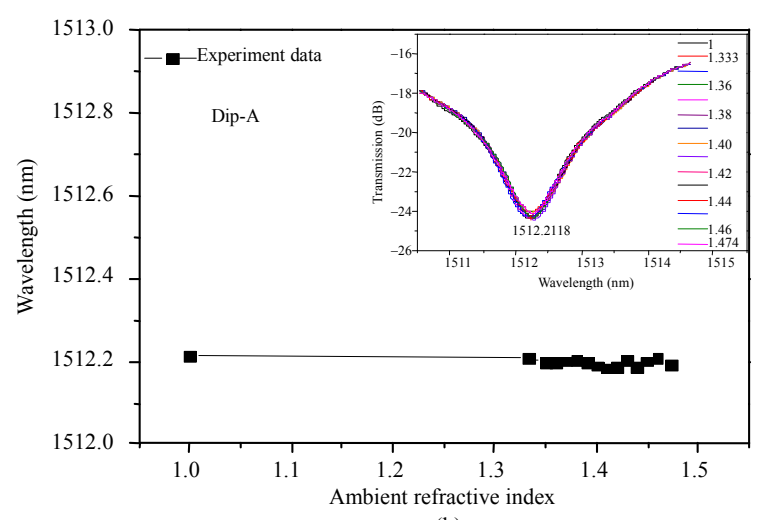

(b)

Fig. 8 Wavelength responses of the MZI to temperature and ARI: (a) wavelength shifts of the $4 \mathrm{~mm}$ long MZI as a function of temperature (triangle: Dip-A; star: Dip-B; solid and dashed lines: results of linear fitting) and (b) wavelength shifts as a function of ARI at Dip-A.

On the other hand, we have also investigated the dependence of the wavelength shift upon ARI which is shown in Fig. 8(b). It can be seen that the dip wavelength is shifted very slightly as ARI increases from 1 to 1.474 , therefore the MZI is believed to be insensitive to ARI. We consider that it is because the interference pattern of the MZI is resulted by 
invoking $\mathrm{LP}_{01}$ and higher order $\mathrm{LP}_{21}$ mode in the core. Those core mode fields are well confined by air holes of PCF. It induces to a result that their effective refractive indices are immune to ARI. At the end the MZI sensor possesses a crucial advantage of ARI insensitivity.

In practical applications, it is beneficial to avoiding cross-sensitivity of temperature in bending measurements. Here in our experiments, both Dip-A and Dip-B show linear and discriminating sensitivities to curvature and temperature, so the structure of the MZI can be used to measure curvature and temperature simultaneously by constructing a matrix consisting of the bending and temperature sensitivities $[7,16]$. The sensing matrix can be determined in (7):

$$
\begin{gathered}
{\left[\begin{array}{c}
\Delta C \\
\Delta T
\end{array}\right]=\left[\begin{array}{ll}
S_{C, A} & S_{T, A} \\
S_{C, B} & S_{T, B}
\end{array}\right]^{-1}\left[\begin{array}{l}
\Delta \lambda_{1} \\
\Delta \lambda_{2}
\end{array}\right]=} \\
{\left[\begin{array}{ll}
0.484 & -0.023 \\
0.246 & -0.016
\end{array}\right]^{-1}\left[\begin{array}{l}
\Delta \lambda_{1} \\
\Delta \lambda_{2}
\end{array}\right] .}
\end{gathered}
$$

\section{Conclusions}

A simple and compact structure of the MZI based on the multimode PCF is proposed by splicing a short section of the PCF between two SMFs with a lateral core-offset. The results show that a dominant interference pattern is generated by $\mathrm{LP}_{01}$ and higher order $\mathrm{LP}_{21}$ mode in the core. Thus, this sensor is not sensitive to ARI and can sense the bending vector to recognize positive and negative directions in one dimension in a large curvature range from $-7.13 \mathrm{~m}^{-1}$ to $7.13 \mathrm{~m}^{-1}$. By constructing a matrix, the curvature and temperature can be measured simultaneously without ARI crosstalk. It indicates that this device has excellent potential for curvature sensing applications.

\section{Acknowledgment}

This work was supported by the National Natural Science Foundation of China (NSFC) (Grant Nos. 61275125, 61308055, and 61307097), Shenzhen Science and Technology Project (Grant
No. JCYJ20140509172609175), and Specialized Research Fund for the Doctoral Program of Higher Education (SRFDP) (Grant No. 20124408120004).

Open Access This article is distributed under the terms of the Creative Commons Attribution License which permits any use, distribution, and reproduction in any medium, provided the original author(s) and source are credited.

\section{References}

[1] H. J. Patrick, C. Chang, and S. T. Vohra, "Long period fiber gratings for structural bending sensing," Electronics Letters, 1998, 34(18): 1773-1775.

[2] Y. Jin, C. Chan, X. Dong, and Y. Zhang, "Temperature-independent bending sensor with tilted fiber Bragg grating interacting with multimode fiber," Optics Communications, 2009, 282(19): 3905-3907.

[3] L. Shao, L. Xiong, C. Chen, A. Laronche, and J. Albert, "Directional bend sensor based on re-crown tilted fiber Bragg grating," Journal of Lightwave Technology, 2010, 28(18): 2681-2687.

[4] M. Deng, C. Tang, T. Zhu, and Y. Rao, "Highly sensitive bend sensor based on Mach-Zehnder interferometer using photonic crystal fiber," Optics Communications, 2011, 284(12): 2849-2853.

[5] D. Monzon-Hernandez, A. Martinez-Rios, I. Torres-Gomez, and G. Salceda-Delgado, "Compact optical fiber curvature sensor based on concatenating two tapers," Optics Letters, 2011, 36(22): 4380-4382.

[6] O. Frazão, J. Viegas, P. Caldas, J. L. Santos, F. M. Araújo, L. A. Ferreira, et al., "All-fiber Mach-Zehnder curvature sensor based on multimode interference combined with a long-period grating," Optics Letters, 2007, 32(21): 3074-3076.

[7] S. Zhang, W. Zhang, S. Gao, P. Geng, and X. Xue, "Fiber-optic bending vector sensor based on Mach-Zehnder interferometer exploiting lateral-offset and up-taper," Optics Letters, 2012, 37(21): 4480-4482.

[8] Z. Ou, Y. Yu, P. Yan, J. Wang, Q. Huang, X. Chen, et al., "Ambient refractive index-independent bending vector sensor based on seven-core photonic crystal fiber using lateral offset splicing," Optics Express, 2013, 21(20): 23812-23821.

[9] J. Zheng, P. Yan, Y. Yu, Z. Ou, J. Wang, X. Chen, et al., "Temperature and index insensitive strain sensor based on a photonic crystal fiber in line Mach-Zehnder interferometer," Optics Communications, 2013, 297(15): 7-11.

[10] Y. Geng, X. Li, X. Tan, Y. Deng, and X. Hong, "Compact and ultrasensitive temperature sensor with 
a fully liquid-filled photonic crystal fiber Mach-Zehnder interferometer," IEEE Sensors Journal, 2013, 14(1): 167-170.

[11] T. Allsop, R. Reeves, D. J. Webb, I. Bennion, and R. Neal, "A high sensitivity refractometer based upon a long period grating Mach-Zehnder interferometer," Review of Scientific Instruments, 2002, 73(4): 1702-1705.

[12] J. Villatoro, V. Minkovich, V. Pruneri, and G. Badenes, "Simple all-microstructured-optical-fiber interferometer built via fusion splicing," Optics Express, 2007, 15(4): 1491-1496.

[13] O. Frazão, S. F. O. Silva, J. Viegas, J. M. Baptista, J.L. Santos, J. Kobelke, et al., "All fiber Mach-Zehnder interferometer based on suspended twin-core fiber," IEEE Photonics Technology Letters,
2010, 22(17): 1300-1302.

[14] B. Dong, J. Hao, and Z. Xu, "Temperature insensitive curvature measurement with a core-offset polarization maintaining photonic crystal fiber based interferometer," Optical Fiber Technology, 2011, 17(3): 233-235.

[15] J. R. Guzman-Sepulveda and D. A. May-Arrioja, "In-fiber directional coupler for high-sensitivity curvature measurement," Optics Express, 2013, 21(10): 11853-11861.

[16] Z. Wu, Y. Liu, Z. Wang, T. Han, S. Li, M. Jiang, et al., "In-line Mach-Zehnder interferometer composed of microtaper and long-period grating in all-solid photonic bandgap fiber," Applied Physics Letters, 2012, 101(14): 141106-1-141106-4. 